\title{
Study on Motion Management of Lung Cancer Treated by CyberKnife: Based on Tumor Position
}

Shenghua Jing ( $\nabla_{\text {jingsh99@139.com ) }}$

East region military command general hospital https://orcid.org/0000-0002-3141-8456

\section{Zhen Wang}

East Region Military Command General Hospital

\section{Changchen Jiang}

East Region Military Command General Hospital

\section{Xiangnan Qiu}

East Region Military Command General Hospital

\section{Taincong Wu}

East Region Military Command General Hospital

\section{Yan Zhang}

East Region Military Command General Hospital

\section{Yikun Li}

East Region Military Command General Hospital

\section{Wei Wang}

Nanjing Medical University

\section{Xiangdong Sun}

East Region Military Command General Hospital

\section{Research Article}

Keywords: lung cancer, tumor motion amplitude, tumor motion management, SRTs, expansion margin

Posted Date: January 7th, 2022

DOI: https://doi.org/10.21203/rs.3.rs-1175811/v1

License: (c) (i) This work is licensed under a Creative Commons Attribution 4.0 International License. Read Full License 


\section{Abstract}

Purpose: We investigated the movement characteristics of lung cancers and the clinical accuracy of tracking lung tumors with Synchrony Respiratory Tracking System (SRTs) during the CyberKnife treatment. We also explored the influencing factors of accuracy. These data provided the appropriate expansion margins of patients with different respiratory characteristics, which was helpful to realize the personalized design of treatment plans of CyberKnife.

Methods and Materials: 73 patients with lung cancer treated with CyberKnife SRTs were selected retrospectively for this study. The patient's age, gender, respiratory characteristics and tumor datas (tumor size, anatomical position and geometric position) were recorded. During treatment, the deviation was checked every $45 \mathrm{~s}$ and compensated by the synchronous respiratory tracking system.

Results: The total mean motion amplitudes and standard deviations of lung tumors in superior-inferior (SI), left-right (LR), and anterior-posterior (AP) directions were $4.15 \pm 3.47 \mathrm{~mm}, 3.98 \pm 3.21 \mathrm{~mm}$ and $3.79 \pm$ $2.73 \mathrm{~mm}$, respectively. The overall mean correlation errors and standard deviations were $0.86 \pm 0.45 \mathrm{~mm}$, $1.04 \pm 0.76 \mathrm{~mm}$ and $0.70 \pm 0.47 \mathrm{~mm}$, respectively. The overall mean prediction errors and standard deviations were $0.18 \pm 0.17 \mathrm{~mm}, 0.35 \pm 0.39 \mathrm{~mm}$ and $0.35 \pm 0.42 \mathrm{~mm}$, respectively. The correlation errors of LR direction were less correlated with the geometric position of the tumor $(r=0.38)$, and not correlated with the anatomical position of the tumor $(r<0.3)$. The prediction errors were moderately correlated with the respiratory amplitude $(r=0.588)$, and less correlated with the baseline drift and the motion amplitude of the tumor ( $r=0.407$ and 0.365 , respectively).

Conclusions: The patient's respiratory amplitude, the tumor motion amplitude, the tumor baseline drift and geometric position were the main factors affecting the tracking accuracy. Tumors at different geometric positions should be treated differently to ensure sufficient dose coverage of the lung tumor target.

\section{Introduction}

A great challenge for lung cancer radiotherapy is how to compensate for the respiratory induced tumor movement. This movement is the main factor hindering the increase of radiotherapy dose, which can occur in all anatomical directions and inhibit the accurate transmission of radiation dose (1). The International Commission on Radiological Units and measurements (ICRU) recommends that the edge of the tumor be added to compensate for the geometric uncertainty caused by this motion and tumor rotation. CyberKnife is widely used in the world as an image-guided real-time tumor tracking therapy (2, 3). The SRTs can compensate for inconsistent respiratory movements and reduce the treatment margins, especially the end expiratory baseline shift. CyberKnife therapy has the characteristics of high fractional dose, short fractional time and high biological dose, but it must have high accuracy and stability $(4,5)$. The edge range of CyberKnife user reports is $2-8 \mathrm{~mm}$, and the common edge range is $3-5 \mathrm{~mm}(6,7)$. 
The accuracy of correlation and prediction in real-time tumor tracking of CyberKnife SRTs is limited (8). Phantom experiments had proved that synchronization can achieve real-time respiratory motion tracking with $0.7 \pm 0.3 \mathrm{~mm}$ accuracy (9). There were many studies on correlation and prediction errors of clinical lung tumor patients. The errors also considered different lobes and different directions (SI, LR and AP). The lung was divided into 9 parts in previous papers, but in this paper, the lung was divided into 27 parts to analyze the motion and errors. This study aims to answer three key clinical questions: 1 . Motion characteristics of lung tumors under free breathing; 2 . The accuracy and related factors of tracking lung tumors by CyberKnife SRT system; 3 . The expansion margin of the lung tumor was guided by the movement characteristics of lung and the tracking accuracy of SRTs $(10,11)$.

\section{Methods And Materials}

\section{Data source}

Data sets from 304 treatment scores of 73 patients with lung cancer (primary or metastatic) were investigated from 2017 to 2020, and these patients had previously been treated with CyberKnife SRTs. The average duration of each data set was $60.6 \mathrm{~min}$. The treatment characteristics of patients were listed in Supplementary Table 1. Before treatment, 3-5 fiducials were implanted into or around the tumor under the guidance of CT or ultrasonic endoscopy. As an internal marker, the fiducial can accurately track moving tumors (12-15). The design of the treatment plan was based on end-inspiratory CT image, and PTV was based on the expansion of GTV by $3 \mathrm{~mm}$ in all directions. The dose to the planning target volume (PTV) was defined as the isodose line of $65-89 \%$, where $100 \%$ was standardized as the maximum dose. The lung tumor movement is defined as the centroid displacement of fiducials relative to the planned position. Some fiducials migrate or rotate during treatment. If the fiducials exceeded the respective anatomical range, the data sets of the subsequent treatment were discarded. The respiratory motion data, lung tumor motion data, correlation error data and prediction error data were extracted from the treatment log files (16).

During respiratory movement, lung tissues at different positions showed inconsistent motion trajectories (17). In order to further study whether there was statistical difference in the accuracy of real-time tracking of lung tumors at different locations by CyberKnife SRTs, the lung tissue was divided into three equal parts along the directions of SI, LR and AP, as shown in Supplementary Figure 1. LR directions were marked as $A, B$ and $C$, SI directions were marked as $D, E$ and F, and $A P$ directions were marked as $G, H$ and I.

\section{Baseline drift}

Three optical markers are used to record external respiratory signals in real time. These markers are optical fiber terminals for transmitting LED signals $(18,19)$. It was reported that the external substitution movement was closely related to the internal tissue movement strongly (20). However, this correlation may change due to a baseline drift of the patient's breathing and gradual relaxation of muscles. Baseline drift means that the slow changes of respiratory baseline in one direction with time (21). Baseline drift 
was calculated by subtracting the absolute value of the lowest point from the highest point of the baseline and dividing by time. Malinowski (22) investigated patients with lung and pancreatic tumors and reported that in $63 \%$ of cases, the relationship between substitutes and tumor location changed.

\section{Correlation and prediction errors}

A pair of orthogonal X-ray tubes was used to take a number of X-ray images of patients. At different stages of the respiratory cycle, the position and direction of several fiducials implanted in or near the tumor were monitored until the SRTS system showed that the respiratory cycle covered $100 \%$. The correlation model will be established between the captured internal fiducial and the external marker data.

The correlation error was defined as the difference between a tumor's location as determined by x-ray images and that calculated by the latest correlation model. If the position given by the model differs from the position indicated by X-ray images by more than $5 \mathrm{~mm}$, the synchronization treatment of CyberKnife will be stopped. The operator will build a new related model by removing all existing data points and acquiring a series of new X-ray images. Multiple synchronization models may be established in a complete treatment. This may be caused by (1) the failure of the CyberKnife synchronization system; (2) the patient asked for rest; and (3) the patient's deep breathing or coughing during CyberKnife synchronization therapy.

Another component of the SRTs is the prediction model, through which the SRTs can obtain the tumor location information 115 milliseconds ahead of time, and the SRTs (23) can complete tumor identification and beam adjustment within 115 milliseconds.

The prediction error was calculated by comparing the predicted location with the actual location after 115 milliseconds. The overall mean errors of each fraction and the standard deviations (SDs) were calculated in SI, LR and AP directions. The Modeler.log file (24) contains the output (modeling points) of the correlation model, which are used to determine the amplitude of motion. The Predictor.log file contains the output of the prediction algorithm (predictor points) and the corresponding modeler points, including the predicted position and prediction error. The ModelPoints.log file contains the time stamp of image acquisition, the central quality of internal markers and the correlation model error for each component of motion and external marker. The Markers.log file records the position of the external markers to analyze the influence of breathing movement on errors. The ERsiData.log file contains the position and status information of the linear accelerator. The ERsiData.log file was used to identify the times when radiation was being delivered, which was used to record the effect of treatment time.

\section{Statistical analysis}

Data of each patient was calculated and expressed as the overall mean \pm standard deviation for all patients. The Pearson correlation coefficient $r$ was evaluated with an uncorrelated test. Comparisons were performed using T-test, and differences were considered significant at the $95 \%$ confidence level $(P<0.05)$. The statistical analyses are based on SPSS statistics of IBM. 
The factors for the tracking accuracy were estimated through the multivariate regression analysis. Correlation errors and prediction errors were specified as dependent variables. Twelve parameters, such as baseline drift, respiratory amplitude, respiratory cycle, treatment time, tumor volume, tumor anatomical position, geometric position, tumor motion amplitude, lung volume, isoline dose, numbers and distance of markers, were extracted as independent variables.

\section{Results}

\section{Respiratory movement and tumor movement characteristics}

For 73 patients, $304 \mathrm{X}$-ray image data sets and 6408 timestamps were analyzed. The average duration of each data set was 60.6 minutes, and the average volume of tracking tumor was $49.99 \pm 50.92 \mathrm{~cm}^{3}$. In Supplementary Table 2, the overall mean and standard deviation of respiratory motion amplitude in $\mathrm{SI}$, $\mathrm{LR}, \mathrm{AP}$ and radial directions were $5.92 \pm 3.11 \mathrm{~mm}, 1.17 \pm 0.68 \mathrm{~mm}$ and $2.57 \pm 1.79 \mathrm{~mm}$, respectively. The overall mean and standard deviation of respiratory motion amplitude in $\mathrm{SI}, \mathrm{LR}, \mathrm{AP}$ and radial directions were $4.15 \pm 3.47 \mathrm{~mm}, 3.98 \pm 3.21 \mathrm{~mm}$, and $3.79 \pm 2.73 \mathrm{~mm}$, respectively. The tumor motion amplitudes of different lobes were shown in Table 1. Single sample t-test data analysis showed that there were significant differences in tumor motion amplitude at different geometric positions $(P=0.001)$. 
Table 1

Overview of the tumor motion amplitudes of different lobes

\begin{tabular}{|c|c|c|c|c|}
\hline \multicolumn{2}{|c|}{ Lung lobes and directions } & \multirow{2}{*}{$\begin{array}{l}\text { Mean }(\mathrm{mm}) \\
5.04\end{array}$} & \multirow{2}{*}{$\begin{array}{l}\mathrm{SD}(\mathrm{mm}) \\
4.13\end{array}$} & \multirow{2}{*}{$\begin{array}{l}\text { Range (mm) } \\
0.64-18.22\end{array}$} \\
\hline RUL & $\mathrm{SI}$ & & & \\
\hline & LR & 3.39 & 2.13 & $0.64-8.39$ \\
\hline & AP & 3.10 & 1.88 & $0.44-8.75$ \\
\hline \multirow[t]{3}{*}{ RML } & $\mathrm{SI}$ & 4.25 & 3.82 & $0.26-15.13$ \\
\hline & LR & 4.72 & 3.62 & $0.23-16.80$ \\
\hline & AP & 5.28 & 3.06 & $0.52-14.76$ \\
\hline \multirow[t]{3}{*}{ RLL } & SI & 4.52 & 2.97 & $0.79-11.75$ \\
\hline & LR & 2.33 & 1.09 & $0.70-4.94$ \\
\hline & AP & 3.13 & 2.58 & $0.57-8.38$ \\
\hline \multirow[t]{3}{*}{ LUL } & $\mathrm{SI}$ & 4.74 & 4.07 & $0.29-13.90$ \\
\hline & LR & 3.12 & 2.48 & $0.94-8.42$ \\
\hline & AP & 2.56 & 1.87 & $0.388-6.856$ \\
\hline \multirow[t]{3}{*}{ LLL } & $\mathrm{SI}$ & 3.28 & 2.29 & $0.30-11.82$ \\
\hline & LR & 4.08 & 2.76 & $0.29-11.06$ \\
\hline & $\mathrm{AP}$ & 3.97 & 2.55 & $0.65-16.90$ \\
\hline
\end{tabular}

The mean tumor motion amplitude in right lung was significantly greater than that in left lung, and the mean tumor motion amplitude in SI direction was significantly greater than that in other directions.

\section{Correlation and prediction errors}

In order to evaluate the errors of CyberKnife SRTs, 304 data sets of 73 patients were analyzed. The correlation and prediction errors of tumors of different lung lobes in SI, LR and AP directions were shown in Table 2. The correlation and prediction errors of different lung lobes and different geometric positions in $\mathrm{SI}, \mathrm{LR}$ and AP directions were shown in Figure 1. Comparing Figure $1(\mathrm{~A})$ and $(\mathrm{B})$, it could be seen that the correlation errors of $\mathrm{AFI}, \mathrm{BFI}, \mathrm{CEI}$ and $\mathrm{CFG}$ positions in SI direction and CEG position in LR direction were higher than the mean correlation errors of corresponding anatomical positions. Similarly, comparing Figure 1 (C) and (D), it could be seen that the prediction errors of $\mathrm{AFI}, \mathrm{BFH}, \mathrm{BFI}$ and CEI positions in $\mathrm{SI}$ direction and AFI position in LR direction were significantly higher than the mean prediction errors of corresponding anatomical positions. 
Table 2

Summary of correlation and prediction errors in 304 fractions

\begin{tabular}{|c|c|c|c|c|c|c|c|}
\hline \multirow{2}{*}{\multicolumn{2}{|c|}{$\begin{array}{l}\text { Lobes and } \\
\text { directions } \\
\text { (Fraction) }\end{array}$}} & \multicolumn{2}{|l|}{ Mean $(\mathrm{mm})$} & \multicolumn{2}{|l|}{$\mathrm{SD}(\mathrm{mm})$} & \multicolumn{2}{|c|}{ Range (mm) } \\
\hline & & $\begin{array}{l}\text { Correlation } \\
\text { error }\end{array}$ & $\begin{array}{l}\text { Prediction } \\
\text { error }\end{array}$ & $\begin{array}{l}\text { Correlation } \\
\text { error }\end{array}$ & $\begin{array}{l}\text { Prediction } \\
\text { error }\end{array}$ & $\begin{array}{l}\text { Correlation } \\
\text { error }\end{array}$ & $\begin{array}{l}\text { Prediction } \\
\text { error }\end{array}$ \\
\hline \multirow{3}{*}{$\begin{array}{l}\text { RUL } \\
(\mathrm{N}=73)\end{array}$} & $\mathrm{SI}$ & 0.85 & 0.14 & 0.34 & 0.11 & $0.37-1.65$ & $0.01-0.47$ \\
\hline & LR & 0.84 & 0.27 & 0.47 & 0.24 & $0.28-2.33$ & $0.03-1.17$ \\
\hline & AP & 0.66 & 0.24 & 0.32 & 0.27 & $0.26-1.85$ & $\begin{array}{l}0.013- \\
1.13\end{array}$ \\
\hline \multirow{3}{*}{$\begin{array}{l}\text { RML } \\
(\mathrm{N}=48)\end{array}$} & $\mathrm{SI}$ & 0.88 & 0.16 & 0.53 & 0.18 & $0.22-2.84$ & $0.02-1.04$ \\
\hline & LR & 1.55 & 0.54 & 1.10 & 0.38 & $0.31-4.36$ & $0.01-1.29$ \\
\hline & $\mathrm{AP}$ & 0.92 & 0.63 & 0.66 & 0.43 & $0.30-4.02$ & $0.04-1.51$ \\
\hline \multirow{3}{*}{$\begin{array}{l}\text { RLL } \\
(\mathrm{N}=41)\end{array}$} & $\mathrm{SI}$ & 0.85 & 0.15 & 0.326 & 0.08 & $0.23-1.59$ & $0.02-0.32$ \\
\hline & LR & 1.158 & 0.17 & 0.69 & 0.12 & $0.40-2.43$ & $0.01-0.36$ \\
\hline & AP & 0.68 & 0.21 & 0.28 & 0.17 & $0.25-1.48$ & $0.03-0.55$ \\
\hline \multirow{3}{*}{$\begin{array}{l}\text { LUL } \\
(\mathrm{N}=44)\end{array}$} & $\mathrm{SI}$ & 1.05 & 0.11 & 0.72 & 0.09 & $0.21-3.79$ & $0.02-0.39$ \\
\hline & LR & 0.55 & 0.11 & 0.29 & 0.07 & $0.15-1.09$ & $0.01-0.24$ \\
\hline & AP & 0.70 & 0.14 & 0.51 & 0.10 & $0.20-2.39$ & $\begin{array}{l}0.010- \\
0.35\end{array}$ \\
\hline \multirow{3}{*}{$\begin{array}{l}\text { LLL } \\
(\mathrm{N}=98)\end{array}$} & $\mathrm{SI}$ & 0.86 & 0.23 & 0.55 & 0.22 & $0.11-3.07$ & $0.02-0.95$ \\
\hline & LR & 1.11 & 0.49 & 0.79 & 0.50 & $0.16-3.78$ & $0.02-2.30$ \\
\hline & AP & 0.68 & 0.44 & 0.48 & 0.53 & $0.10-2.73$ & $0.01-2.55$ \\
\hline \multirow{3}{*}{$\begin{array}{l}\text { Whole } \\
\text { lung } \\
(\mathrm{N}=304)\end{array}$} & $\mathrm{SI}$ & 0.86 & 0.18 & 0.50 & 0.17 & $\begin{array}{l}0.11- \\
3.789\end{array}$ & $0.01-1.04$ \\
\hline & LR & 1.04 & 0.35 & 0.76 & 0.38 & $0.15-4.36$ & $0.01-2.30$ \\
\hline & AP & 0.70 & 0.34 & 0.47 & 0.42 & $0.09-4.02$ & $0.01-2.55$ \\
\hline
\end{tabular}

As it could be seen from Figure 1 (C), except for RML and LLL (the mean prediction errors were slightly greater than $0.5 \mathrm{~mm})$, the mean prediction errors of tumors in other locations were very small $(0.2 \mathrm{~mm})$. However, it could be seen from Figure 1 (D) that only the tumors located in the geometric positions of AFI, $\mathrm{BFH}, \mathrm{BFI}, \mathrm{CEI}$ and $\mathrm{CFG}$ had high prediction errors in SI and LR directions (among which, the average prediction errors of tumors located in the geometric positions of AFI were as high as $2 \mathrm{~mm}$ in SI and LR directions), while the mean prediction errors of tumors located in other geometric positions were very small. 
The overall mean correlation and prediction errors of different lung lobes in all directions were shown in Table 3. From the overall statistical data, the mean and standard deviation of prediction errors were far less than the correlation errors. The errors of the lower lobe of lung were larger than that of the upper lobe of lung, whether the correlation errors or prediction errors.

Table 3

Statistical tables of correlation, prediction and total error based on different lung lobes

\begin{tabular}{|c|c|c|c|c|c|c|c|c|}
\hline \multirow[t]{2}{*}{ Lobes } & \multirow[t]{2}{*}{ Directions } & \multicolumn{2}{|c|}{$\begin{array}{l}\text { Correlation error } \\
(\mathrm{mm})\end{array}$} & \multicolumn{2}{|c|}{$\begin{array}{l}\text { Prediction error } \\
(\mathrm{mm})\end{array}$} & \multirow[t]{2}{*}{$\begin{array}{l}\text { Targeting error } \\
(\mathrm{mm})\end{array}$} & \multicolumn{2}{|c|}{$\begin{array}{l}\text { Total } \\
\text { error(mm) }\end{array}$} \\
\hline & & $95 \% \mathrm{Cl}$ & $99 \% \mathrm{Cl}$ & $95 \% \mathrm{Cl}$ & $99 \% \mathrm{Cl}$ & & $\begin{array}{l}95 \% \\
\mathrm{Cl}\end{array}$ & $\begin{array}{l}99 \% \\
\mathrm{Cl}\end{array}$ \\
\hline \multirow[t]{3}{*}{ RUL } & SI & 1.19 & 1.53 & 0.25 & 0.36 & 0.5 & 1.94 & 2.39 \\
\hline & LR & 1.31 & 1.78 & 0.5 & 0.75 & 0.5 & 2.31 & 3.03 \\
\hline & AP & 0.99 & 1.31 & 0.51 & 0.78 & 0.5 & 2 & 2.59 \\
\hline \multirow[t]{3}{*}{ RML } & SI & 1.41 & 1.94 & 0.34 & 0.52 & 0.5 & 2.25 & 2.96 \\
\hline & LR & 2.65 & 3.75 & 0.92 & 1.3 & 0.5 & 4.07 & 5.55 \\
\hline & AP & 1.58 & 2.24 & 1.06 & 1.49 & 0.5 & 3.14 & 4.23 \\
\hline \multirow[t]{3}{*}{ RLL } & SI & 1.18 & 1.51 & 0.23 & 0.31 & 0.5 & 1.91 & 2.32 \\
\hline & LR & 1.85 & 2.54 & 0.29 & 0.41 & 0.5 & 2.64 & 3.45 \\
\hline & AP & 0.96 & 1.24 & 0.38 & 0.55 & 0.5 & 1.84 & 2.29 \\
\hline \multirow[t]{3}{*}{ LUL } & SI & 1.77 & 2.49 & 0.2 & 0.29 & 0.5 & 2.47 & 3.28 \\
\hline & LR & 0.84 & 1.13 & 0.18 & 0.25 & 0.5 & 1.52 & 1.88 \\
\hline & AP & 1.21 & 1.72 & 0.24 & 0.34 & 0.5 & 1.95 & 2.56 \\
\hline \multirow[t]{3}{*}{ LLL } & SI & 1.41 & 1.96 & 0.45 & 0.67 & 0.5 & 2.36 & 3.13 \\
\hline & LR & 1.91 & 2.7 & 0.99 & 1.49 & 0.5 & 3.4 & 4.69 \\
\hline & AP & 1.16 & 1.64 & 0.97 & 1.5 & 0.5 & 2.63 & 3.64 \\
\hline
\end{tabular}

\section{Uncertainty influencing factors}

There are many factors that affect the uncertainty, including individual differences of patients (respiratory frequency and respiratory amplitude), location of the tumor (anatomic and geometric), tumor motion amplitude, tumor motion speed, baseline drift, respiratory phase shift, the tumor volume and treatment time. The Pearson correlation analysis was used to analyze the correlation between 9 factors and errors. The results of correlation analysis were summarized in Supplementary Table 3. The scatter plot of the correlation between uncertainty factors and correlation errors (Figure 2) and prediction errors (Figure 3 ) in SI, LR and AP directions were shown in Figure 2 and 3, respectively. 
It could be seen from Figure 2 that the correlation error in LR direction was less correlated with the geometric position of the tumor $(r=0.38)$, and not correlated with the anatomical position of the tumor $(r$ $<0.3$ ). It could be seen from Figure 3 that the prediction error was moderately correlated with respiratory amplitude $(r=0.588)$, and less correlated with baseline drift and tumor motion amplitude $(r=0.407$ and 0.365 , respectively).

\section{Tumor expansion margins}

In addition to clinical requirements, target accuracy, the correlation error and prediction error need to be considered in the tumor expansion boundary of SRTs treatment. Target accuracy refers to the difference between the expected target and the place where the CyberKnife actually provides radiation. In the montly QA inspection of our CyberKnife treatment center, the target accuracy was $0.50055 \mathrm{~mm}$. This target accuracy varies based on the monthly QA inspection of each treatment center.

Two standard deviations from the mean value of each anatomical direction were used to ensure $95 \%$ coverage of modeling points. Similarly, three standard deviations from the mean value of each anatomical direction were used to ensure $99 \%$ coverage of modeling points. However, if the minimum or maximum deviation was less than two standard deviations, they were replaced. The total errors in SI, LR and AP directions of different lung lobes (Table 3 ) and different tumor locations (Table 4) were shown in Table 3 and 4. 
Table 4

Statistical tables of correlation, prediction and total error based on different positions

\begin{tabular}{|c|c|c|c|c|c|c|c|c|}
\hline \multirow[t]{2}{*}{ Position } & \multirow[t]{2}{*}{ Directions } & \multicolumn{2}{|c|}{$\begin{array}{l}\text { Correlation error } \\
(\mathrm{mm})\end{array}$} & \multicolumn{2}{|c|}{$\begin{array}{l}\text { Prediction error } \\
(\mathrm{mm})\end{array}$} & \multirow[t]{2}{*}{$\begin{array}{l}\text { Targeting error } \\
(\mathrm{mm})\end{array}$} & \multicolumn{2}{|c|}{$\begin{array}{l}\text { Total } \\
\text { error(mm) }\end{array}$} \\
\hline & & $95 \% \mathrm{Cl}$ & $99 \% \mathrm{Cl}$ & $95 \% \mathrm{Cl}$ & $99 \% \mathrm{Cl}$ & & $\begin{array}{l}95 \% \\
\mathrm{Cl}\end{array}$ & $\begin{array}{l}99 \% \\
\mathrm{Cl}\end{array}$ \\
\hline \multirow[t]{3}{*}{ AFI } & SI & 2.35 & 2.82 & 2.23 & 2.41 & 0.5 & 5.08 & 5.73 \\
\hline & LR & 0.3 & 0.46 & 2.0 & 2.17 & 0.5 & 2.8 & 3.13 \\
\hline & AP & 0.7 & 0.84 & 0.78 & 0.93 & 0.5 & 1.98 & 2.27 \\
\hline \multirow[t]{3}{*}{ BFH } & SI & 1.37 & 1.54 & 0.74 & 0.91 & 0.5 & 2.61 & 2.95 \\
\hline & LR & 0.83 & 1.01 & 0.72 & 0.88 & 0.5 & 2.05 & 2.39 \\
\hline & AP & 1.14 & 1.39 & 0.18 & 0.26 & 0.5 & 1.82 & 2.15 \\
\hline \multirow[t]{3}{*}{$\mathrm{BFI}$} & SI & 1.68 & 2.11 & 0.82 & 0.97 & 0.5 & 3 & 3.58 \\
\hline & LR & 1.13 & 1.43 & 0.62 & 0.72 & 0.5 & 2.25 & 2.65 \\
\hline & AP & 1.11 & 1.37 & 0.24 & 0.32 & 0.5 & 1.85 & 2.19 \\
\hline \multirow[t]{3}{*}{ CEG } & SI & 1.32 & 1.69 & 0.36 & 0.43 & 0.5 & 2.18 & 2.62 \\
\hline & LR & 2.15 & 2.41 & 0.41 & 0.51 & 0.5 & 3.06 & 3.42 \\
\hline & AP & 1.56 & 1.95 & 0.59 & 0.70 & 0.5 & 2.65 & 3.15 \\
\hline \multirow[t]{3}{*}{ CEI } & SI & 2.32 & 2.76 & 0.84 & 1.16 & 0.5 & 3.66 & 4.42 \\
\hline & LR & 0.68 & 0.95 & 0.82 & 1.04 & 0.5 & 2 & 2.49 \\
\hline & $\mathrm{AP}$ & 0.77 & 0.95 & 0.18 & 0.33 & 0.5 & 1.45 & 1.78 \\
\hline \multirow[t]{3}{*}{ CFG } & SI & 1.83 & 2.04 & 0.61 & 0.78 & 0.5 & 2.94 & 3.32 \\
\hline & LR & 0.75 & 1.01 & 0.63 & 0.71 & 0.5 & 1.88 & 2.22 \\
\hline & AP & 0.97 & 1.23 & 0.39 & 0.47 & 0.5 & 1.86 & 2.20 \\
\hline \multirow[t]{3}{*}{ Others } & SI & 1.55 & 1.93 & 0.21 & 0.28 & 0.5 & 2.26 & 2.71 \\
\hline & LR & 1.03 & 1.38 & 0.32 & 0.41 & 0.5 & 1.85 & 2.29 \\
\hline & AP & 1.23 & 1.40 & 0.44 & 0.52 & 0.5 & 2.17 & 2.42 \\
\hline
\end{tabular}

\section{Discussion}

Considering the individual differences of patients' respiratory characteristics, this paper first studied whether the patients' respiratory amplitude and respiratory rate were related to the uncertainty errors. The overall mean and standard deviation of respiratory motion amplitude in SI, LR, AP and radial directions 
were $5.92 \pm 3.11 \mathrm{~mm}, 1.17 \pm 0.68 \mathrm{~mm}$ and $2.57 \pm 1.79 \mathrm{~mm}$, respectively. The Pearson correlation analysis showed that there was a significant correlation between respiratory amplitude in SI direction and lung volume $(P=0.005)$. This showed that there were significant differences in the respiratory amplitude of different patients in SI direction. And the respiratory amplitude of patients was moderately correlated with the prediction error. Therefore, individualized tumor expansion margin is necessary for Cyberknife SRTs treatment.

The baseline drift of the patient's breathing was also studied in this paper. The relationship between baseline drift and respiratory cycle (A), respiratory amplitude (B), treatment time (C) in SI, LR and AP directions were shown in Figure 4. The baseline drift was less correlated with the treatment time $(r=$ 0.435). And when the treatment time was between 55 and 65 minutes, the baseline drift was the largest. Therefore, the best treatment time was less than 55 minutes.

Malinowski Kathleen et al. (25) pointed out that the tumor motion amplitude has an important influence on tracking accuracy. Pepin E W et al. (26) pointed out that there was no strong correlation between tumor location, size and expansion margins, but we got inconsistent results. According to the Pearson correlation coefficient $r$, it was founded that the correlation error in LR direction was less correlated with the geometric position of the tumor $(r=0.38)$, and not correlated with the anatomical position of the tumor $(r<0.3)$. It could be seen from Figure 4 that the prediction error was moderately correlated with respiratory amplitude $(r=0.588)$, and less correlated with baseline drift and tumor motion amplitude $(r=$ 0.407 and 0.365 , respectively).

There are some limitations in this paper. First, 304 treatment data sets of 73 patients with lung cancer were divided into 27 parts (an average of 11 data sets per part), and the sample size was slightly lower. However, the data in this paper showed that the tumor geometric position, the respiratory amplitude, the tumor motion amplitude and baseline drift were significantly correlated with the clinical accuracy of CyberKnife SRTs in the treatment of lung cancer.

Second, the relationship among errors and prediction models and motion is based on external LED markers. The correlation and prediction models were constructed based on external LED signals through SRT system. Therefore, the location of LED may affect the model errors. However, it is difficult to extract specific parameters from each patient's LED marker data, because CyberKnife treatment lasted longer than IMRT. In addition, each patient in this study can breathe freely during CyberKnife treatment. Although these abnormal data have been excluded from statistics, there were some irregular breathing patterns in the respiratory data.

\section{Conclusions}

With regard to the margins of tumor expansion, some research showed that (27) the tumor margin was 7 $\mathrm{mm}$ in SI direction, and $5 \mathrm{~mm}$ in LR and AP directions. If the coverage rate of modeling points reaches $99 \%$, LR and AP should be extended to 6-7mm. Hoogeman M et al. (16) pointed out that the mean correlation error was less than $0.3 \mathrm{~mm}$, and the mean prediction error was $0 \mathrm{~mm}$. The correlation and 
prediction errors were highly correlated with the respiratory motion amplitude. The research of Hoogeman $M$ et al. was theoretical values, and there was no research on how high the accuracy can be achieved in actual clinical patients. Inoue $\mathrm{M}$ et al. (28) pointed out that the tumor tracking accuracy was related to the tumor motion amplitude in SI direction and the tumor motion speed in SI and AP directions. Our results were consistent with them and supplemented. According to this paper, we founded that the correlation error was correlated with tumor geometric position, and the prediction error was moderately correlated with respiratory amplitude, baseline drift and tumor motion amplitude.

Our results provide detailed information as accurate as possible for expansion margins of lung tumor in different location. The respiratory amplitude, tumor motion amplitude, baseline drift and the tumor geometric position were the main factors affecting the correlation and prediction errors. According to the characteristics and location of the tumor, selecting the appropriate expansion margin is of great significance to improve the accuracy of CyberKnife in the treatment of lung cancer. It should be noted that the CT scan images of the treatment plan in this study are based on the end of inspiration rather than the end of expiration. In order to understand the results of this study, we need to distinguish the differences between the two methods.

\section{Declarations}

\section{Ethics approval and consent to participate}

Not applicable.

\section{Consent for publication}

Not applicable.

\section{Availability of data and materials}

All data generated or analysed during this study are included in this published article [and its supplementary information files].

\section{Competing interests}

The authors declare that they have no competing interests.

\section{Funding}

No. 


\section{Authors' contributions}

* S.H.J. and Z.W. contributed equally to this work.

SH J designed the study and wrote the manuscript. Z W, CC J and XN Q specially collected clinical data. YK L and $Y Z$ used statistics to analyze and integrate research data. Thanks to TC $W$ and $W W$ for supervising the manuscript research. Thanks to XD S for the feasibility analysis of the research conclusions and other data.

All authors approved the version to be published; and all authors have agreed to be accountable for all aspects of the work in ensuring that questions related to the accuracy or integrity of any part of the work are appropriately investigated and resolved.

\section{Acknowledgements}

Not applicable.

\section{References}

1. Marants, Raanan, Vandervoort, Eric, Cygler, Joanna E. Evaluation of the 4D RADPOS dosimetry system for dose and position quality assurance of CyberKnife. Med Phys. 2018; doi: 10.1002/mp.13102

2. Nuyttens, Joost Jan, van de Pol, Marjan. The CyberKnife radiosurgery system for lung cancer. Expert Rev Med Devices. 2012; 9 (5): 465-75. doi: 10.1586/erd.12.35

3. Vlaskou Badra, E, Baumgartl, M, Fabiano, S, et al. Stereotactic radiotherapy for early stage non-small cell lung cancer: current standards and ongoing research. Transl Lung Cancer Res. 2021; 10 (4): Transl Lung Cancer Res. doi: 10.21037/tlcr-20-860

4. Akino, Yuichi, Sumida, Iori, Shiomi, Hiroya, et al. Evaluation of the accuracy of the CyberKnife Synchrony ${ }^{\mathrm{TM}}$ Respiratory Tracking System using a plastic scintillator. Med Phys. 2018; doi:

10.1002/mp.13028

5. Yihang Guo, Hongqing Zhuang, Lujun Zhao. Influence of different image-guided tracking methods upon the local efficacy of CyberKnife treatment in lung tumors [J]. Thoracic Cancer, 2015: 255-259. doi: 10.1111/1759-7714.12174

6. Yang, ZY, Chang, Y, Liu, HY, et al. Target margin design for real-time lung tumor tracking stereotactic body radiation therapy using CyberKnife Xsight Lung Tracking System. Sci Rep. 2017; 7 (1): Sci Rep. doi: 10.1038/s41598-017-11128-w

7. Liu, M, Cygler, JE, Vandervoort, E. Geometrical tracking accuracy and appropriate PTV margins for robotic radiosurgery of liver lesions by SBRT. Acta Oncol. 2019; 58 (6): Acta Oncol. doi: 10.1080/0284186X.2019.1578896 
8. Liang, Zhiwen, Liu, Hongyuan, Xue, Jun, et al. Evaluation of the intra- and interfractional tumor motion and variability by fiducial-based real-time tracking in liver stereotactic body radiation therapy. J Appl Clin Med Phys. 2018; 19 (3): 94-100. doi: 10.1002/acm2.12292

9. Dieterich, Sonja, Pawlicki, Todd. Cyberknife image-guided delivery and quality assurance. Int $J$ Radiat Oncol Biol Phys. 2008; 71 (1): S126-30. doi: 10.1016/j.ijrobp.2007.08.081

10. Yang, Zhi-Yong, Chang, Yu, Liu, Hong-Yuan, Liu, Gang, Li, Qin. Target margin design for real-time lung tumor tracking stereotactic body radiation therapy using CyberKnife Xsight Lung Tracking System. Sci Rep. 2017; 7 (1): 10826. doi: 10.1038/s41598-017-11128-w

11. Floriano, Alejandro, García, Rafael, Moreno, Ramon, et al. Retrospective evaluation of CTV to PTV margins using CyberKnife in patients with thoracic tumors. J Appl Clin Med Phys. 2014; 15 (6): 4825. doi: $10.1120 /$ jacmp.v15i6.4825

12. Mongeon, M., Filion, E., Gilbert, P., et al. ["Retrospective evaluation of endovascular fiducial markers insertion in cyberknife stereotactic radiotherapy treatment planning of lung neoplasms"] J Vasc Interv Radiol. 2014; 25 (3): S192-S193. doi: 10.1016/j.jvir.2013.12.519

13. Shimizu, Shinichi, Osaka, Yasuhiro, Shinohara, Nobuo, et al. Use of implanted markers and interportal adjustment with real-time tracking radiotherapy system to reduce intrafraction prostate motion. Int J Radiat Oncol Biol Phys. 2011; 81 (4): e393-399. doi: 10.1016/j.jijobp.2011.04.043

14. Nakayama, Masao, Nishimura, Hideki, Mayahara, Hiroshi, et al. Clinical log data analysis for assessing the accuracy of the CyberKnife fiducial-free lung tumor tracking system. Pract Radiat Oncol. 2017; 8 (2): e63-e70. doi: 10.1016/j.prro.2017.10.014

15. Van den Ende, RPJ, Kerkhof, EM, Rigter, LS, et al. Feasibility of Gold Fiducial Markers as a Surrogate for Gross Tumor Volume Position in Image-Guided Radiation Therapy of Rectal Cancer. Int J Radiat Oncol Biol Phys. 2019; 105 (5): Int J Radiat Oncol Biol Phys. doi: 10.1016/j.jirobp.2019.08.052

16. Hoogeman M, Jean-BriacPrévost, Nuyttens $J$, et al. Clinical Accuracy of the Respiratory Tumor Tracking System of the CyberKnife: Assessment by Analysis of Log Files[J]. International Journal of Radiation Oncology Biology Physics, 2009, 74(1):297-303.

17. Dhont, J, Vandemeulebroucke, J, Burghelea, $M$, et al. The long- and short-term variability of breathing induced tumor motion in lung and liver over the course of a radiotherapy treatment. Radiother Oncol. 2018; 126 (2): Radiother Oncol. doi: 10.1016/j.radonc.2017.09.001

18. Kord, M, Kluge, A, Kufeld, M, et al. Risks and Benefits of Fiducial Marker Placement in Tumor Lesions for Robotic Radiosurgery: Technical Outcomes of 357 Implantations. Cancers (Basel). 2021; 13 (19): Cancers (Basel). doi: 10.3390/cancers13194838

19. Ferris, William S, Kissick, Michael W, Bayouth, John E, Culberson, Wesley S, Smilowitz, Jennifer B, (2020). Evaluation of radixact motion synchrony for 3D respiratory motion: Modeling accuracy and dosimetric fidelity. J Appl Clin Med Phys, 21 (9), 96-106. https://doi.org/10.1002/acm2.12978

20. Pettersson, N, Oderinde, OM, Murphy, J, et al. Intrafractional relationship changes between an external breathing signal and fiducial marker positions in pancreatic cancer patients. J Appl Clin Med Phys. 2020; 21 (3): J Appl Clin Med Phys. doi: 10.1002/acm2.12841 
21. Akino, Yuichi, Shiomi, Hiroya, Sumida, lori, et al. Impacts of respiratory phase shifts on motiontracking accuracy of the CyberKnife Synchrony ${ }^{\text {TM }}$ Respiratory Tracking System. Med Phys. 2019; 46 (9): 3757-3766. doi: 10.1002/mp.13523

22. Malinowski K, McAvoy TJ, George R, Dietrich S, D'Souza WD. Incidence of changes in respirationinduced tumor motion and its relationship with respiratory surrogates during individual treatment fractions. Int J Radiat Oncol Biol Phys. 2012; 82:1665-1673.

23. Zhang, Jianping, Wang, Lin, Li, Xiaobo, Huang, Miaoyun, Xu, Benhua, (2021). Quantification of Intrafraction and Interfraction Tumor Motion Amplitude and Prediction Error for Different Liver Tumor Trajectories in Cyberknife Synchrony Tracking. Int J Radiat Oncol Biol Phys, 109 (5), 1588-1605. https://doi.org/10.1016/j.ijrobp.2020.11.036

24. Nakayama, $\mathrm{M}$, Nishimura, $\mathrm{H}$, Mayahara, $\mathrm{H}$, et al. Clinical log data analysis for assessing the accuracy of the CyberKnife fiducial-free lung tumor tracking system. Pract Radiat Oncol. 0; 8 (2): Pract Radiat Oncol. doi: 10.1016/j.prro.2017.10.014

25. Malinowski, Kathleen, McAvoy, Thomas J, George, Rohini, et al. Incidence of changes in respirationinduced tumor motion and its relationship with respiratory surrogates during individual treatment fractions. Int J Radiat Oncol Biol Phys. 2012; 82 (5): 1665-1673. doi: 10.1016/j.ijrobp.2011.02.048

26. Pepin, Eric W, Wu, Huanmei, Zhang, Yuenian, et al. Correlation and prediction uncertainties in the cyberknife synchrony respiratory tracking system. Med Phys. 2011; 38 (7): 4036-4044. doi: 10.1118/1.3596527

27. Okawa, K, Inoue, M, Sakae, T. Development of a tracking error prediction system for the CyberKnife Synchrony Respiratory Tracking System with use of support vector regression. Med Biol Eng Comput. 2021; 59 (1112): Med Biol Eng Comput. doi: 10.1007/s11517-021-02445-4

28. Inoue $\mathrm{M}$, Okawa $\mathrm{K}$, Taguchi $\mathrm{J}$, et al. Factors affecting the accuracy of respiratory tracking of the image-guided robotic radiosurgery system[J]. Japanese Journal of Radiology, 2019, 37(10):727-734.

\section{Figures}




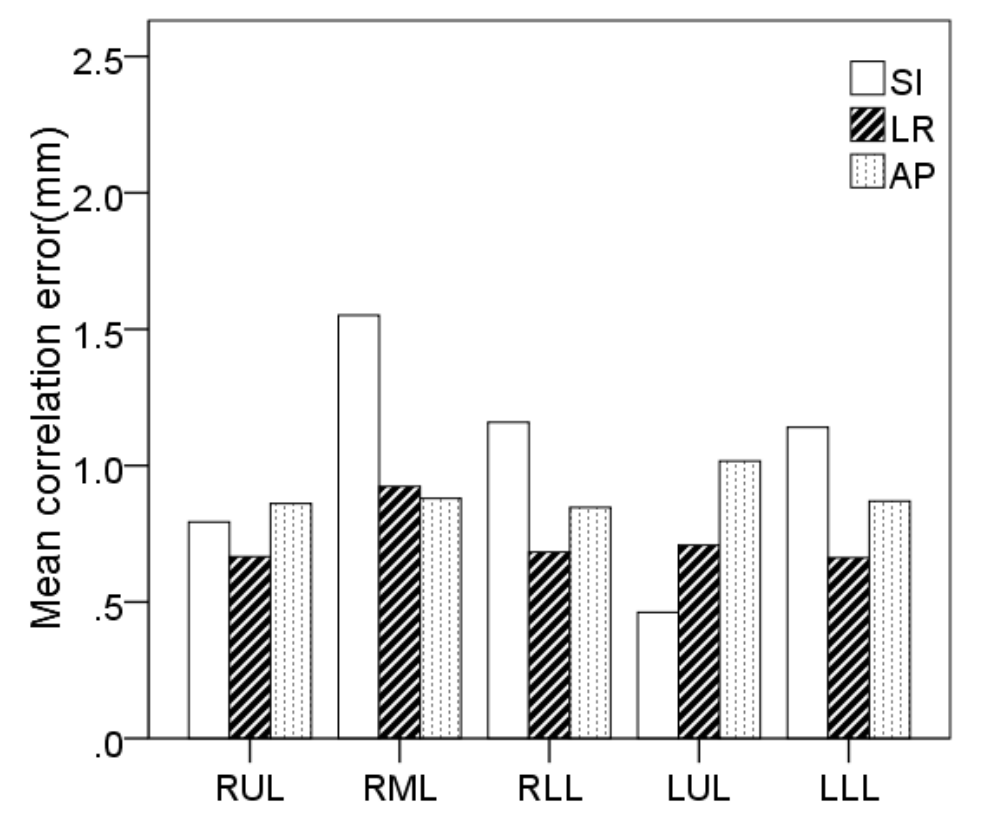

A. Anatomical location

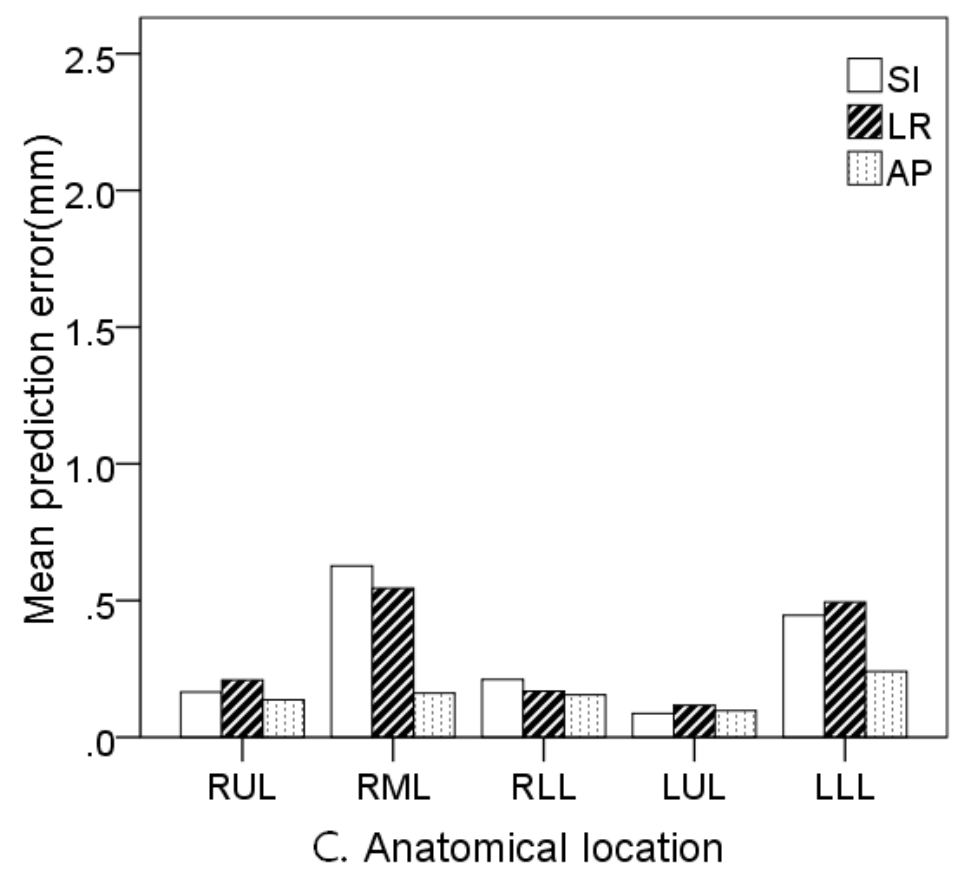

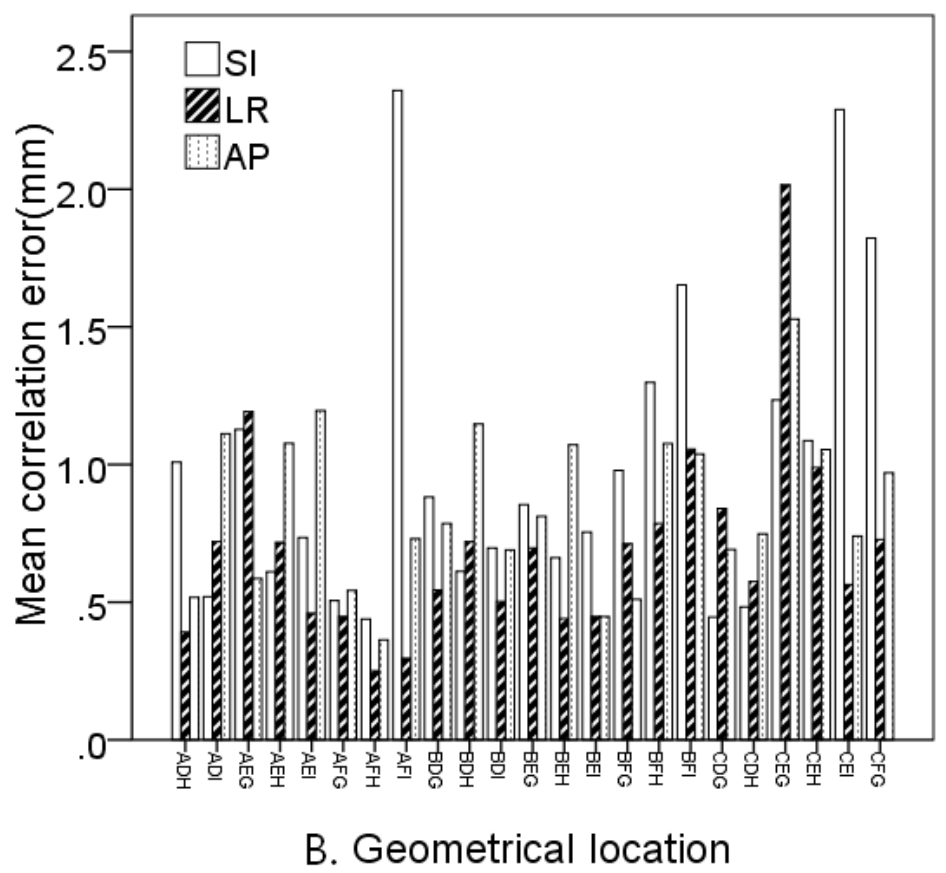

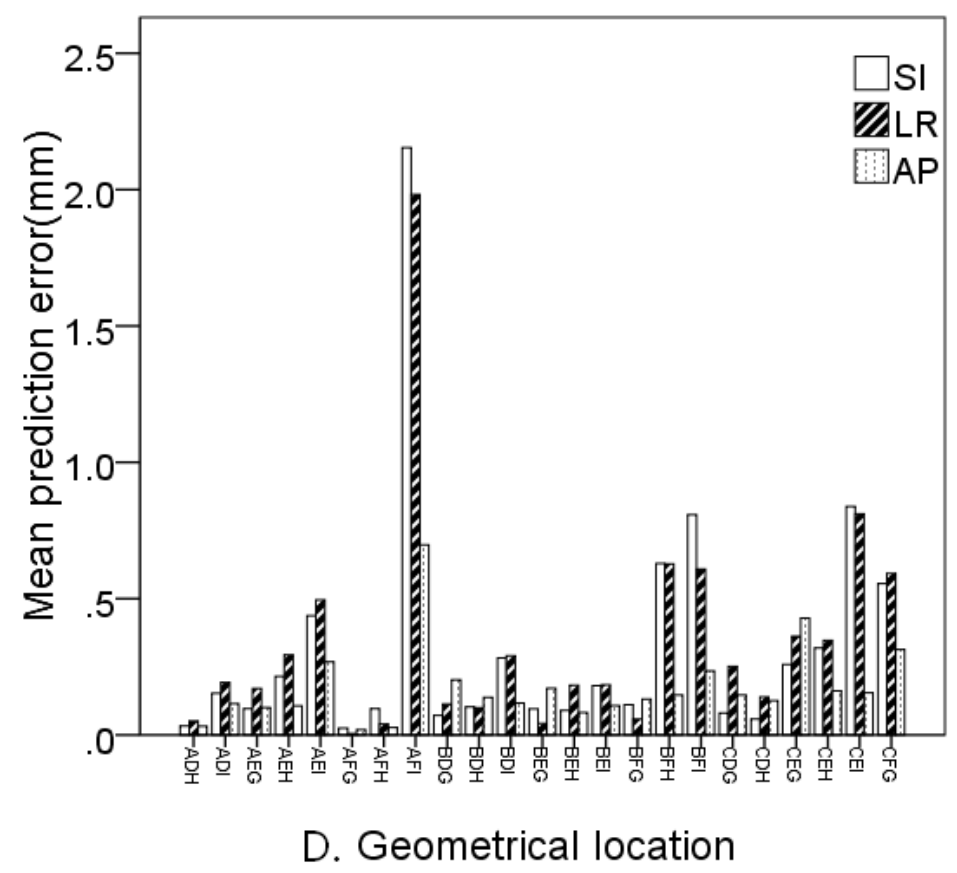

Figure 1

The correlation and prediction errors of different lung lobes and different geometric positions in SI, LR and AP directions were shown in Figure 1. 

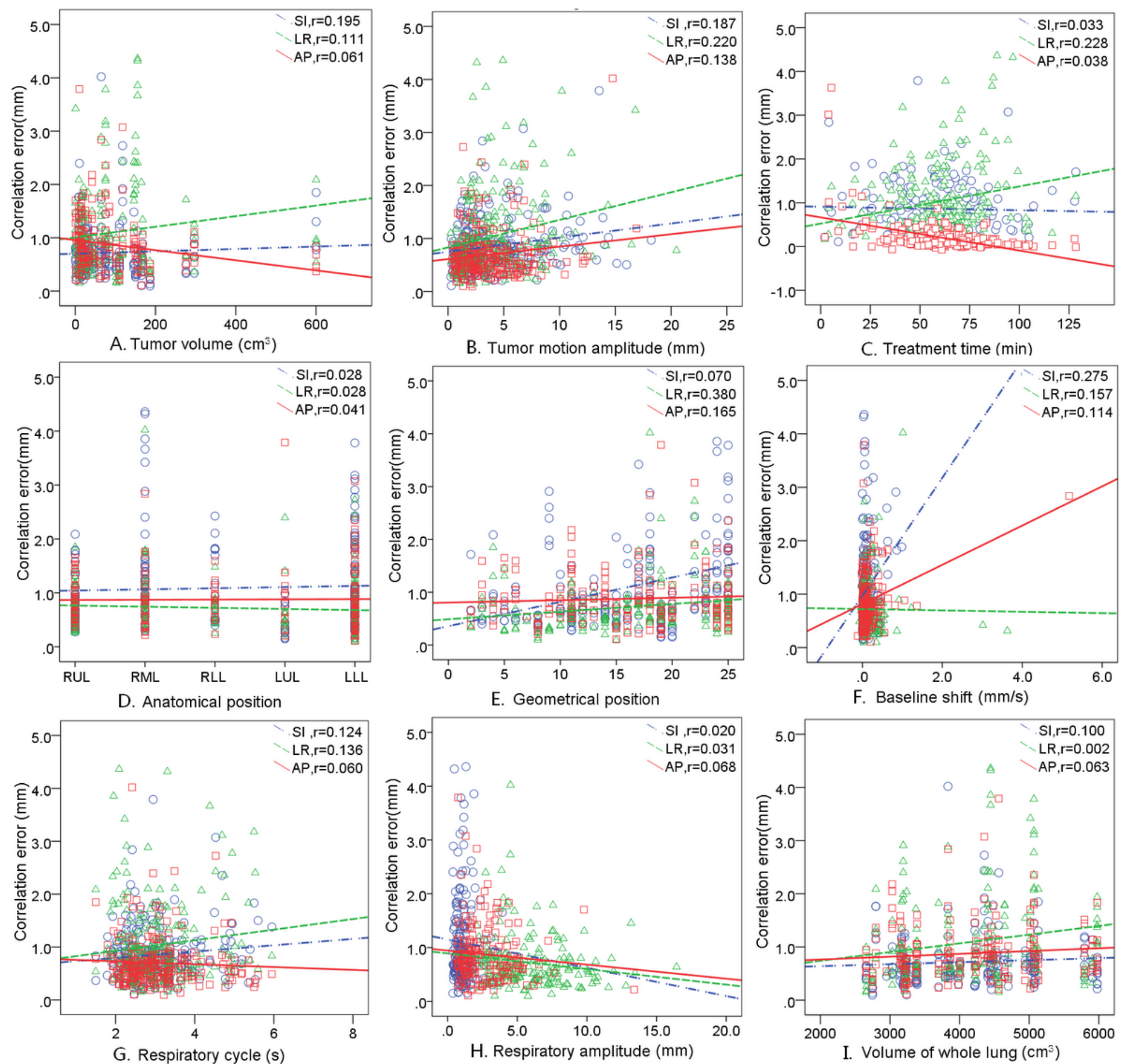

Figure 2

The scatter plot of the correlation between uncertainty factors and correlation errors in SI, LR and AP directions were shown in Figure 2 and 3, respectively. 

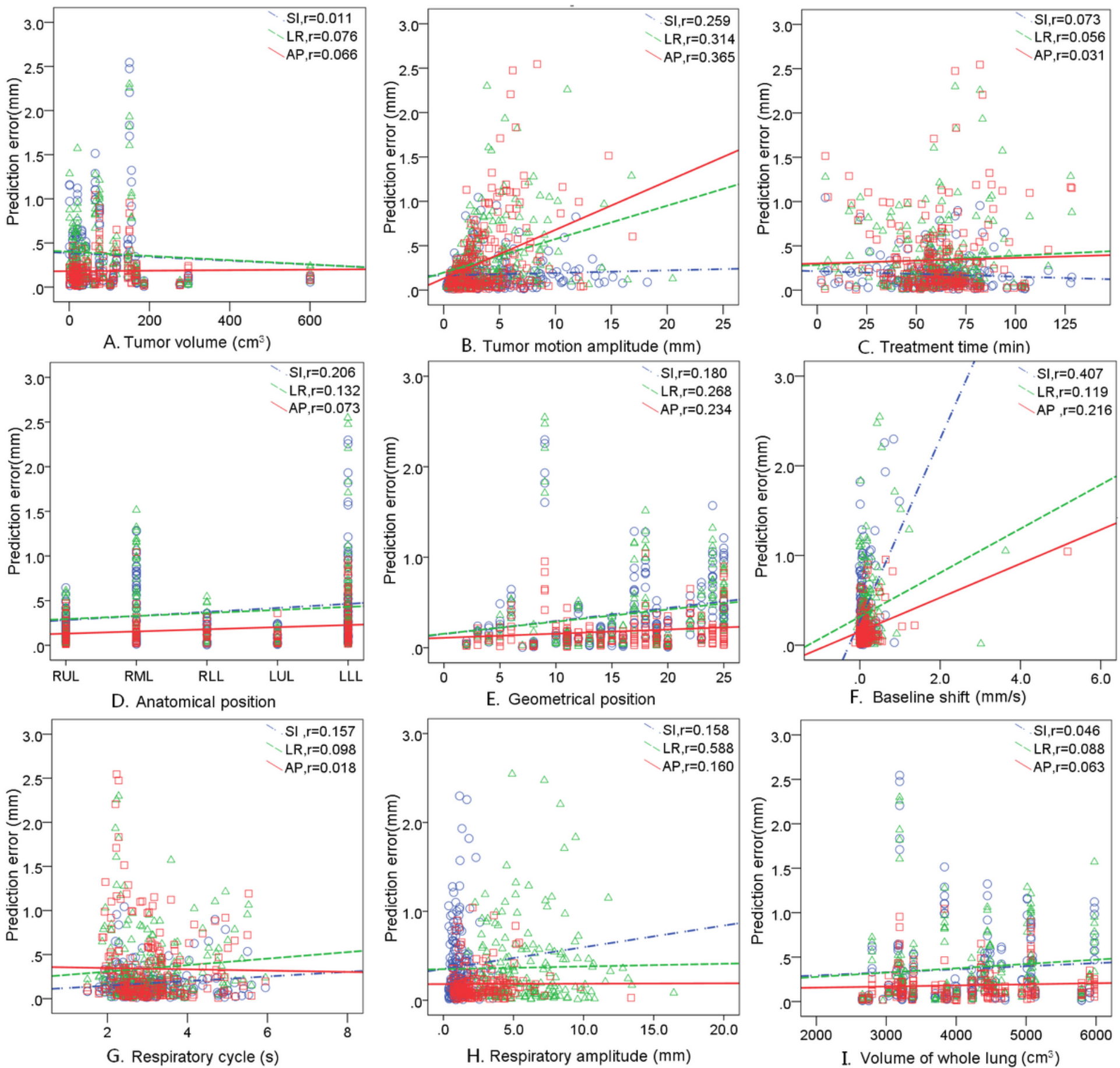

Figure 3

The scatter plot of the correlation between uncertainty factors and prediction errors (Figure 3) in SI, LR and AP directions were shown in Figure 2 and 3, respectively. 

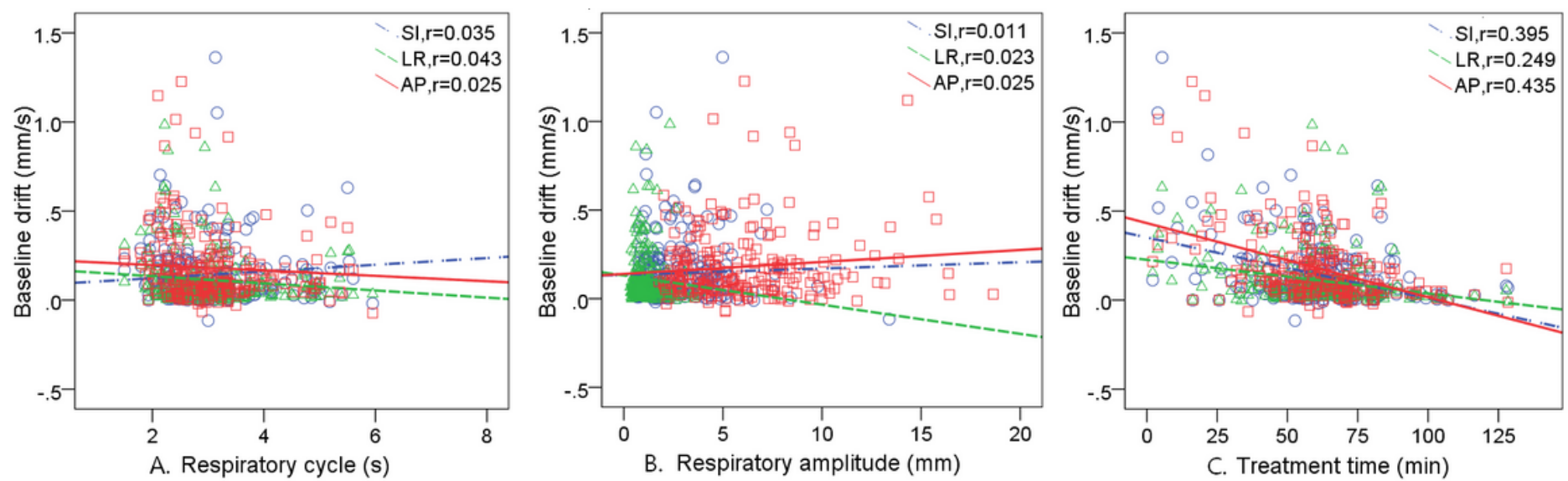

\section{Figure 4}

The baseline drift of the patient's breathing was also studied in this paper. The relationship between baseline drift and respiratory cycle (A), respiratory amplitude (B), treatment time (C) in SI, LR and AP directions were shown in Figure 4.

\section{Supplementary Files}

This is a list of supplementary files associated with this preprint. Click to download.

- SupplementaryFigure1.png

- SupplementaryTables.docx 